\title{
Child attention to pain and pain tolerance are dependent upon anxiety and attention
}

\section{control: An eye-tracking study}

Heathcote, L.C. ${ }^{1}$, MSc, Eccleston, C. ${ }^{2}$, Phd, Lau, J. ${ }^{3}$, Phd, Mueller, S. ${ }^{4}$, Phd, Fox, E. ${ }^{1}$, Phd, Bosmans, $\mathrm{M}^{4}$., MSc \& Vervoort, T. ${ }^{4}$, Phd*

1 Department of Experimental Psychology, University of Oxford, United Kingdom

2 Centre for Pain Research, University of Bath, United Kingdom

3 Department of Psychology, King's College London, London, United Kingdom

4 Department of Experimental-Clinical and Health Psychology, Ghent University, Belgium

* Corresponding author: Tine Vervoort, Department of Experimental-Clinical and Health Psychology, Ghent University, Henri Dunantlaan 2, B- 9000 Ghent, Belgium. Tel: +32 (0)9 2649108 Fax: +32 (0)9 26464 71. Electronic mail may be sent to Tine.Vervoort@Ugent.be

Lauren Heathcote is a DPhil student at the University of Oxford, a Research Training Fellow for UK charity Action Medical Research for Children (Grant Reference: GN2122), and a trainee member of Pain in Child Health, a research training initiative of the Canadian Institutes of Health Research. Tine Vervoort is a Postdoctoral fellow of the Fund for Scientific Research - Flanders (Belgium) (F.W.O.).

\section{Category: Original article}

Number of text pages:

Number of tables:

Number of Figures:

Keywords: attention, eye tracking, facial pain expression, pain catastrophizing, anxiety, attention control, dual process model, children/adolescents, avoidance behaviour 


\section{ABSTRACT}

This study examined how anxiety-related variables and attention control interacted to predict children's attention to personally relevant pain cues using eye-tracking methodology, and their pain tolerance on the cold pressor test (CPT). Thirty-seven children aged 8-17 years viewed photographs of other children displaying painful facial expressions during the CPT, before completing the CPT themselves. Findings indicated that anxiety and attention control did not impact their initial fixations on pain or neutral faces, but did impact how long they dwelled on pain versus neutral faces. Specifically, anxiety showed opposite relationships with dwell time depending on levels of attention control. For children reporting low levels of attention control, higher anxiety was associated with decreasing dwell time on pain faces as opposed to neutral faces. Conversely, for children reporting high levels of attention control, higher anxiety was associated with increasing dwell time on pain faces as opposed to neutral faces. Anxiety likewise showed opposite relationships with pain tolerance and anticipation of pain in the cold pressor task depending on whether children reported low or high levels of attention control. For children with low attention control, increasing anxiety was associated with anticipating more pain and tolerating pain for less time, and the opposite pattern was observed for children with high attention control. This is the first study to examine children's attention to pain cues using eye-tracking technology in the context of a salient painful experience. Data suggest attention control is an important moderator of anxiety on multiple outcomes relevant to young people’s pain experiences. 


\section{INTRODUCTION}

Affective-motivational models propose that pain imposes an attentional priority and motivates avoidance behaviour, particularly when one is anxious about pain $[2,15,19,49]$. Relatedly, how one attends to pain, and subsequently engages in behaviour to avoid pain, is thought critical in the transition from acute to chronic pain. As pain often first becomes chronic in youth, understanding the relationship between anxiety and pain attending, as well as avoidance behaviour, is particularly important in paediatric populations [27]. Paediatric research in this area is only beginning, yet both paediatric and adult literatures are marked by conflicting findings, with some studies showing that increased anxiety contributes to more pain attending and behavioural avoidance [13,14,24,42], whilst others provide null findings $[34,36]$, or evidence counter to expectations $[8,43,44]$. Understanding when anxiety contributes to these outcomes is important for identifying those at risk for adverse pain experiences.

Attention control, the ability to focus effortfully in the face of distraction and flexibly shift attention, is likely important in understanding the variable relationship between painrelated anxiety and pain attending. Indeed, we recently found that adolescents who catastrophize about pain, and have low attention control, showed increasing vigilance to pain facial expressions [23]. However, whilst attention control provides one explanation for mixed findings, our results are preliminary, and outstanding questions remain.

Specifically, most studies have relied on paradigms that examine static measures of attention, indirectly via response times (e.g., dot-probe [6,9,47]). Eye-tracking technology can alternatively provide continuous measures of attention over time. This may be particularly relevant in the context of effortful attention control, which is likely to have more pronounced effects on attention maintenance as opposed to initial selection. Further, most studies have relied on linguistic stimuli (pain words), which have low ecological validity and are indirectly 
related to pain. A significant advance would be to use pictorial stimuli that relate to personally relevant impending pain (e.g., see [47]).

There are also conceptual considerations. Specifically, catastrophizing has been studied as the primary index of pain-related anxiety. However, affective-motivational models $[2,15,19,49]$ posit that general anxiety symptoms when facing pain also influence attention. Moreover, preliminary evidence suggests that catastrophizing in childhood may be best understood as anxiety about pain rather than catastrophic beliefs [20]. Therefore, examining broader anxiety symptoms, as well as catastrophizing, in children's attending to pain is important. Further, while preliminary findings suggest attention control is important in understanding variable relationships between anxiety and attention, it remains unknown whether it has a similar moderating impact on behavioural avoidance, which is considered key in explaining pain outcomes.

The present study examines the impact of anxiety, catastrophizing, and attention control upon child/adolescent attention to personally relevant pain cues, measured using eyetracking methodology, as well as how these variables impact tolerance for cold pressor pain. Following previous studies, we suggest that low tolerance for cold pressor pain can be usefully thought of as avoidance of further pain, and thus indicative of avoidance behaviour (see e.g., [8,47]). We hypothesized that, particularly when attention control is low, anxiety and pain catastrophizing will contribute to (1) increased attending towards pain cues, and (2) decreased tolerance of pain.

\section{METHODS}

\section{1 $\underline{\text { Participants }}$}

The majority of the participants were recruited from a sample of schoolchildren and adolescents (hereafter referred to as 'children') $(N=462)$ who participated in a questionnaire 
study about pain experiences that took place approximately 5 months previously (unpublished data). Only children who had provided informed consent to be re-contacted were approached $(N=136)$. Exclusion criteria for the current study were as follows: (1) recurrent or chronic pain, (2) developmental delay, (3) having insufficient knowledge of the Dutch language, and (4) not being between the ages of 8-17 years. From the total of 136 children, 112 were contacted via their parents (the remaining did not provide contact details $(N=10)$, or were younger or older than 8-17 years $(N=14))$. Of those contacted, $96.4 \%(N=108)$ met the inclusion criteria and $41.07 \%(N=46)$ agreed to participate. Thirty-two percent $(N=36)$ refused to participate. The main reason for refusal to participate was "lack of time" or "other family responsibilities". The remainder of the children $(N=21)$ showed initial interest in the study but were not contactable beyond the first time of contact (i.e., did not respond to a subsequent email or voicemail). To account for potential data loss during eye-tracking and to increase sample size, an additional 9 children were recruited via social media advertisement (advertisement was aimed at parents). There were no differences in anxiety, pain catastrophizing, and attention control between participants recruited via schools and those recruited via social media (all $t(52) \leq|1.63|$, ns). As one of the participants recruited from the previous questionnaire study was used as a pilot subject, the final sample of the present study consisted of 54 children (range 8-17 years, $M=12.1$ years, $S D=2.39,19$ boys). The parents of these children also participated in a study that occurred directly following the current study. The parental study examined the relationship between parent attention flexibility and emotion regulatory processes in response to their child's pain. Procedures relevant to the parental study occurred following the currently described methodology and are thus not expected to interfere with current results. Participants were compensated $30 €$ for participating in this study. The study was approved by the Faculty of Psychology and Educational Sciences ethics committee, Ghent University, Belgium. 


\section{$\underline{2.2 \text { Apparatus }}$}

A cold pressor apparatus was used as an experimental pain task to induce pain in participants and to measure pain tolerance. Participants were instructed to lower their right hand into the cold water up to just above the wrist. Participants maintained their hand in the cold pressor box up to their pain tolerance level or an uninformed ceiling of 4 minutes. The temperature of the water was maintained at $10^{\circ} \mathrm{C}\left(+/-1^{\circ} \mathrm{C}\right)$ and was circulated continuously by a pump (see also [47]). The cold pressor apparatus is well suited for use with children and the pain experienced is considered to provide an analogue for various naturally occurring acute pains $[3,7]$. A second tank was also used, for which the water was maintained at room temperature $\left(21^{\circ} \mathrm{C} ;+/-1^{\circ} \mathrm{C}\right)$. To standardize skin temperature prior to cold pressor immersion, all participants first immersed their hand in the room temperature water tank for a total of 2 minutes (see also [7,47]. This procedure will henceforth be referred to as the Cold Pressor Test (CPT).

\section{$\underline{2.3 \text { Pain tolerance }}$}

Pain tolerance was defined as the participant's total time of immersion in the cold water in seconds, measured by a stopwatch. Prior to the cold pressor test, participants were provided with the following instructions 'Hold your hand in the cold water as long as you can endure the pain. When you think/feel 'I cannot endure the pain any longer - I cannot handle more', I want you to say 'STOP' and take your arm out of the cold water box'. The maximum duration of exposure to the cold pressor test was 4 minutes. However, the participants were not informed of this ceiling so that there would be no risk that they mistakenly thought they were expected to leave their arm in the water for this length of time.

\section{$\underline{2.4 \text { Self-report measures }}$}

Attention control

Attention control was assessed with the Dutch version of the Attention Control Scale 
(ACS) [31]. The ACS consists of 20 items, and yields a total score that can range from 20 to 80, with higher scores indicating good attention control. The ACS has two subscale scores for attention focusing (e.g., "My concentration is good even if there is music in the room around me”) and attention shifting (e.g., "It is easy for me to alternate between two different tasks"). The ACS has shown both good reliability and predictive utility, predicting resistance to interference in Stroop-like spatial conflict tasks as well as attentional disengagement from threat stimuli among highly anxious people [18]. Attention control has also been measured with good reliability and validity in children [23,29]. Cronbach alpha in this study was .67 for the total score.

\section{State anxiety}

State anxiety was assessed by the State version of the Dutch State-Trait Anxiety Inventory for Children [4,40]. The STAIC-state is a 20-item questionnaire designed to measure current anxious state in children. Participants are presented with various emotions (e.g., calm, nervous) and asked to read each item and to decide how they feel "right now." Each item consists of a statement beginning “I feel....", and ending with three possible emotional states (e.g., 'very calm', 'calm' or 'not calm'). Total scores can range from 0 to 40. The decision to measure state rather than trait anxiety was based on previous findings indicating that state anxiety is usually highly correlated with trait anxiety and is likely to be a more proximal predictor of participants’ situational responses (see also [48]). Furthermore, we were interested in state anxiety symptoms in the face of an upcoming painful experience (the cold pressor test), making the measure relevant within the context of pain. The STAIC-state has been shown to be a reliable and valid instrument in previous research $($ see $[4,40])$. Cronbach alpha in this study was .74.

Catastrophic thinking about pain 
A situation-specific measure based upon the original Pain Catastrophizing Scale for Children (PCS-C; [12]) was administered to measure the participants' catastrophizing thoughts about the upcoming pain task. Again, we were interested in state catastrophizing in the face of an upcoming painful experience (the cold pressor test), making the measure relevant for this healthy sample, and more relevant for the outcome of pain tolerance. The original PCS-C consists of 13 items describing different thoughts and feelings that young people may experience when they are in pain, and yields three subscale scores for rumination, magnification, and helplessness. The PCS-C has shown to be a reliable and valid instrument in young people from 9 to 15 years [12]. The use of a situation-specific measure of catastrophizing, i.e., assessing the level of catastrophizing thoughts about an upcoming pain task, is consistent with previous studies [45]. The situation-specific measure of the PCS-C consists of 2 adapted items from each subscale (Rumination: "At this moment, to what extent do you keep thinking about how much pain you will experience during the test?”; Magnification: "At this moment, to what extent do you think that, because of the pain, something serious might happen to you?"; Helplessness: “At this moment, to what extent do you think you will not be able to endure the test because of the pain?"). The 6 items of the situation-specific PCS-C were rated on an 11-point numerical rating scale ( 0 = 'not at all'; 10 = 'very much'), and were completed before the attention task (i.e., eye-tracking task). The total score on catastrophizing could range from 0 to 60 and was used as an index of the participant's catastrophizing thoughts about anticipated pain. Cronbach alpha in this study was 81 .

Anticipated and experienced pain intensity

Anticipated and experienced pain intensity during the cold pressor test were assessed using 11-point 1-item scales with the anchors 'no pain' (0) and 'a lot of pain' (10). Participants were requested to provide written ratings for these measures immediately before 
and after completing the cold pressor test. For the current study, the participant's self-reported anticipated and experienced pain intensity were considered secondary outcome measures (pain tolerance was the primary outcome of interest).

\section{$\underline{2.5 \text { Stimulus materials }}$}

The stimulus set for the viewing task consisted of 40 grey-scaled pictures of 10 different children (5 boys and 5 girls; age range 9-16 years) displaying neutral and pain facial expressions. These pictures were previously used in a dot-probe study in children and an eyetracking study in parents examining child and parental attention to child pain, respectively (see $[47,48]$ ). These pictures were previously reliably coded for occurrence and intensity of facial pain display by means of the Child Facial Coding System (CFCS) [10]. For each of the 10 children in the stimulus set, 4 pictures were chosen to reflect each of 4 categories of facial pain expression: one picture of a particular child depicted the child's neutral face (NFE); one picture depicted the child with a low pain expression (LFE); one showed a moderate pain expression (MFE), and one depicted a high pain expression (HFE). Using the 40-picture set, three types of picture pairings were generated, resulting in 30 slides. Specifically, each slide consisted of two pictures of the same child presenting a neutral expression combined with the same child's low pain, moderate pain, or high pain expression. This allowed examination of whether participants' selective attention to pain faces varied with varying levels of facial pain expressiveness. Pairs were compiled twice such that the neutral expression appeared equally often on the left and right side (i.e., 60 slides were shown: 20 neutral-low pain; 20 neutralmoderate pain, and 20 neutral-high pain). The validity of the present stimulus set is supported by previous findings that differential facial pain expressiveness (i.e., NFE, MFE, LFE, HFE) reflects differences in observers' pain intensity ratings such that increasing levels of facial pain expressiveness corresponded with observers’ increasing pain ratings (see [46,47]).

\section{$\underline{2.6 \text { Eye movement measurement }}$}


Eye movements were tracked with a $300 \mathrm{~Hz}$ Tobii (TX300) table-mounted eye tracker (Tobii Technology AB - , Danderyd, Sweden). This system consists of a 17-inch computer screen with a camera and infrared LED optics embedded beneath it. The system records eye movements based on the corneal reflection caused by the infrared light source. Participants were seated comfortably $60 \mathrm{~cm}$ away from the centre of the screen. In order to calibrate the eye-tracker, participants were asked to focus on nine sequentially appearing red dots presented in random placement on the screen. The viewing task commenced after valid calibration.

\subsection{Viewing (eye-tracking) task}

Participants were informed that they would complete a viewing task, in which their eye movements would be tracked. Participants were informed that during the task, they would view photographs of other children on the screen. To make the stimuli salient and related to a personal painful experience, participants were told that these photographs represented children undergoing the pain procedure that they would subsequently perform (i.e., the cold pressor test). Participants were shown an overview of one trial (on paper) in order to ensure familiarity with the experimental set up. Participants were instructed to first focus on a centrally-presented white fixation cross when it appeared on screen and then to simply view the faces that would subsequently appear on the screen. Each trial within the viewing task began with a 500 ms presentation of the white fixation cross. Then, a slide with the pair of facial stimuli against a grey background was presented for $3500 \mathrm{~ms}$ and participants were free to visually explore the slide. Following an inter-trial interval of $200 \mathrm{~ms}$ (grey screen), the next trial again began with the presentation of the fixation cross. In total, the experiment consisted of 60 trials: each of the 30 slides was presented twice, once with the pain face on the left and once on the right side of the screen. Pictures were $16 \mathrm{~cm}$ high and $10 \mathrm{~cm}$ wide. Pictures were 
separated by $4.4 \mathrm{~cm}$ from their central points. Slides were presented to participants in a fully randomized order.

\section{$\underline{2.8 \text { Picture pain intensity ratings }}$}

After completing the viewing task, participants were asked to rate each presented pain face for pain intensity using a 0-10 NRS (i.e., 'How much pain has the child displayed on the picture; anchors; 'no pain at all'; ‘a lot of pain'). Pictures were presented on a computer screen using Office PowerPoint in a randomized order that was fixed across participants. Participants were instructed to make written ratings of pain intensity for all pictures and were encouraged to proceed as fast as possible. Picture ratings were averaged for each level of facial pain display (NFE, LFE, MFE, HFE) resulting in 4 mean pain intensity ratings ranging from 0-10. This allowed examining whether differences in facial expressiveness of the pictures matched differences in the participant pain intensity ratings of the pictures.

\subsection{Procedure}

All participants were invited by telephone and received standardized information about the study. When parents and their children provided consent, they were invited to the laboratory at Ghent University where the study was conducted (In July 2014 and August/September 2014). Upon arrival at the laboratory, one of two experimenters (both female) accompanied the parent and their child to the test-room. They were were told that the child would perform a computerized eye-tracking task (ie, the viewing task), and complete a number of questionnaires. Furthermore, the cold pressor apparatus was shown to the parent and their child, and they were told that the child could remove their arm from the water at any time, for any reason, and that the child could withdraw from participation at any time.

After obtaining written parental and child consent, experimenter 1 stayed with the participant in the test-room while experimenter 2 accompanied the parent to an adjacent room. Before performing the viewing task, participants were requested to fill out measures on 
attention control, state anxiety, and state pain catastrophizing. Participants then completed the viewing (ie, eye-tracking) task. As noted above, to make the stimuli salient and related to a personal painful experience, participants were told that these photographs represented children undergoing the pain procedure that they would subsequently perform (i.e., the cold pressor test). Following the viewing task, the participant's pain tolerance was assessed during the cold pressor test. Participants' anticipated and experienced pain for and during the cold pressor test was assessed before and after they completed the task, respectively. Participants and parents could not see or hear each other throughout the duration of the study procedure. In addition, to prevent any contact between the experimenter and the participant, the experimenter was seated silently behind a screen during performance of the cold pressor test. After completion of the entire study procedure, parent-child dyads were reunited in the testroom and were fully debriefed about the purpose of the study.

\section{$\underline{2.10 \text { Eye movement parameters and data analysis }}$}

Gaze behaviour was analyzed off-line with the Tobii software analysis package (Tobii Studio 3.2) using the Identification Velocity-Threshold (I-VT) filter (classifier: 30\%/s; Velocity calculator window length: $20 \mathrm{~ms}$ ). The minimum fixation duration was set at $100 \mathrm{~ms}$. The I-VT fixation classifier is based on the Velocity-Threshold identification fixation filter as described by Salvucci and Goldberg [37] and Komogortsev et al [28]. Within the present study, the two target pictures were defined as areas of interest (AOIs) within which eye movements would be monitored. Two eye movement parameters were calculated: (1) probability of first fixation, and (2) total fixation duration. Probability of first fixation refers to whether or not participants first fixated their attention on the pain face or the neutral face. A 'first fixation bias' refers to a higher probability of the participant to first fixate on the pain face rather than the neutral face. Accordingly, first fixation bias was calculated by computing the number of trials when the first fixation was directed on the pain face as a proportion of all 
trials in which the first fixation was made to either face (proportion scores $>0.5$ reflect an orienting bias toward pain faces; $0.5=$ no bias; $<0.5$ reflect an orienting bias toward neutral faces). This was done separately for each facial pain expression category (LFE, MFE, HFE), resulting in 3 probability indices of first fixation bias. Total fixation duration for each facial expression was generated by averaging the total time participants spent looking at faces for each facial expression category separately (NFE, LFE, MFE, HFE). A 'total fixation bias' refers to when a participant spends longer looking at pain faces rather than neutral faces. In the current study (as in previous studies), total fixation duration may also be referred to as “dwell time”.

To examine the impact of participant anxiety, pain catastrophizing, and attention control upon attention to pain, a series of repeated measures analyses of covariance (ANCOVA) were performed for each of the eye movement indices (i.e., probability of first fixation and total fixation duration) with facial expressiveness as a within subject factor and attention control and anxiety or pain catastrophizing entered as covariates, respectively, in separate analyses. We did not add anxiety and pain catastrophizing into the same analyses due to insufficient power to detect potential four-way interactions. To examine the impact of anxiety, pain catastrophizing, and attention control upon pain tolerance, univariate analyses of covariance (ANCOVAs) were performed with pain tolerance as the dependent variable and attention control and anxiety or pain catastrophizing entered as covariates, respectively, in separate analyses. Additional analyses were also performed with anticipated and experienced pain intensity during CPT performance as the dependent variable, as this was considered a secondary outcome within the present study.

Continuous predictor variables (anxiety/pain catastrophizing/attention control) were centered prior to entering the analyses. Centering reduces the multicollinearity between predictors and any interaction terms among them and facilitates post-hoc probing of 
significant interaction effects [26]. Further, in case the analyses with total fixation duration revealed significant interactions with facial expressiveness, bias indices ('total fixation bias') were calculated to further aid interpretation of direction of effects. Separate total fixation bias scores were calculated for each level of facial pain expressiveness (HFE, MFE, LFE). In these analyses, positive values of total fixation bias (i.e., mean total dwell time on pain faces subtracted from mean total dwell time on neutral faces) indicated that dwell time on pain faces was higher than dwell time on neutral faces, whereas a negative score indicated the reverse: higher dwell time on neutral faces. ANCOVA was performed on these bias indices.

In case of significant interactions, additional moderation analyses were performed to interpret the interaction effects (i.e., whether the association between the predictor variable and the outcome was significant only for high levels of the moderator variable, low levels of the moderator variable, or both). All moderation analyses followed the procedure outlined by Holmbeck et al. [26], i.e., two new conditional continuous moderator variables were computed by (1) subtracting 1 SD from the centered moderator variable (i.e., high attention control ) and (2) adding 1 SD to the centered moderator variable (i.e., low attention control). Next, two additional ANCOVAs were performed - incorporating each of these new conditional continuous moderator variables - to test the significance for high $(+1 \underline{\mathrm{SD}}$ above the mean) and low (-1 $\underline{\mathrm{SD}}$ below the mean) values of the conditional centered moderator variable. Whenever the sphericity assumption was violated (Mauchly’s test of sphericity was $p<.05$ ), Greenhouse-Geisser corrections (with adjusted degrees of freedom, or NDf) were performed.

\section{RESULTS}

\section{$\underline{3.1 \text { Participant characteristics }}$}


17 participants were discarded from analyses due to sub-optimal overall gaze track status (i.e., eye movements tracked less than $70 \%$ of total task viewing time). This is in line with previous eye-tracking studies [46]. The final sample entered in the analyses reported below therefore consisted of 37 children (14 boys) aged 8-17 years $(M=12.1$ years, $S D=2.45)$. Those participants who were discarded did not differ from the participants retained in the analysis on any of the independent or dependent measures (all $t(52) \leq|1.58|$, ns). Mean scores, standard deviations, and correlations between measures are reported in Table 1 . The mean level of state anxiety $(M=12.2 ; S D=5.48)$, pain catastrophizing $(M=15.8 ; S D=10.0)$, and attention control $(M=33.3 ; S D=6.06)$ in the present sample are comparable with those obtained in previous samples of school youths $[4,31,45]$. Participants reported moderate levels of anticipated $(M=4.05 ; S D=2.21)$ and experienced mean pain intensity $(M=5.00 ; S D=2.05)$ during CPT performance. Participants’ CPT immersion time ranged from 18 to 240 seconds with a mean of $124.4 \mathrm{~s}(S D=99.1)$. Participants' pain tolerance in the current study is therefore in line with previous studies [47]. About two third of the participants (59.5\%) withdrew their hand from the cold water before the four minute ceiling was reached.

Pearson correlation analyses indicated that self-report measures and pain tolerance did not correlate significantly with participant age (all $r \leq|.05|$, ns). There were no significant differences between girls and boys for any of the measures (all $t(35) \leq|1.00|$, ns), except for anticipated and experienced pain intensity which was higher amongst girls (both $t(35) \leq-2.22$, $p<.05)$. State anxiety was significantly correlated with state pain catastrophizing $(r=.41$, $p<.05)$. State anxiety and state pain catastrophizing were not significantly correlated with attention control ( $r=-.12$ and -.20 , respectively, ns), and none of these measures were correlated with pain tolerance (all $r \leq|.27|$, ns). However, both the participant's anticipated and experienced pain intensity during CPT were significantly correlated with state pain 
catastrophizing (both $r \geq .38, p<.05$ ). Experienced pain intensity was also significantly negatively correlated with pain tolerance $(r=-.34, p<.05)$.

\section{- INSERT TABLE 1 ABOUT HERE -}

\section{$\underline{3.2}$ Picture ratings}

In order to examine whether the different picture categories of facial pain expressiveness matched differences in the participant's ratings of pain intensity (therefore to confirm stimulus categories), participants' pain intensity ratings of the pictures were examined using a repeated measures ANOVA. Results revealed significant differences between ratings of the four expression levels $(F(3,34)=309.4, \epsilon=.66 ; \operatorname{NDf}(1.98 ; 22.44)$, $p<.0001)$. Differences were in the expected direction. Specifically, contrasts revealed that high expressive pain faces ( $M=68.4 S D=15.6)$ were rated significantly higher than moderate expressive pain faces $(M=55.4 ; S D=14.5 ; F(1,36)=160.66, p<.0001)$ which, in turn, were rated significantly higher than low expressive pain faces $(M=44.4 ; S D=14.9 ; F(1,36)=82.6$, $p<.0001)$ which, in turn, were rated significantly higher than neutral faces $(M=29.0 ; S D=15.9$; $F(1,36)=243.4, p<.0001)$. Adding either anxiety or pain catastrophizing, respectively, as a covariate to the repeated measures ANOVA revealed no main effects or interactions effects (all $F(1,35) \leq .2 .40, \mathrm{~ns})$.

3.3 The impact of anxiety and pain catastrophizing upon attention to pain, and the moderating $\underline{\text { role of attention control }}$

\subsubsection{Probability of first fixation}

We first investigated whether or not participants first fixated on pain faces significantly more than neutral faces (i.e., whether or not there was an overall first fixation bias). As a primary repeated measures ANOVA revealed that there were no significant 
differences between first fixation bias towards low $(M=.53)$, medium $(M=.53)$, and high expressive pain faces $(M=.52)(F(2,35)=.07$, ns), we calculated a mean first fixation bias score across all three pain expressions. A one-sampled t-test revealed a mean first fixation bias $(M=.53)$ significantly greater than chance (.50) indicating that participants first fixated significantly more on pain faces than neutral faces $(t(36)=3.30, \mathrm{p}<.005)$.

To investigate the impact of anxiety and the moderating role of attention control upon the participant's first fixation bias, a repeated measures ANCOVA was performed with anxiety and attention control entered as covariates. Analysis indicated no main or interaction effects for anxiety, attention control, and/or facial expressiveness (all $F \leq 1.06$, ns).

The analysis examining the impact of pain catastrophizing and the moderating role of attention control revealed no main or interaction effects for pain catastrophizing, attention control, and/or facial expressiveness (all $F \leq 1.43$, ns).

\subsubsection{Total fixation duration}

We first investigated whether or not participants dwelled longer on pain faces compared to neutral faces (i.e., whether or not there was an overall total fixation bias to pain). A repeated measures ANOVA with facial expressiveness as a within-subject factor (i.e., dwell time on neutral, low pain, moderate pain, and high pain faces) revealed a significant effect $(F(3,34)=17.17 ; \epsilon=.61 ; \operatorname{NDf}(1.83 ; 20.74), p<.0001)$. Contrasts revealed that dwell time on pain faces significantly increased with each level of pain expressiveness (i.e., $M=_{\text {TFD_lowpain }}=1.28 \mathrm{~s}$; $M={ }_{\text {TFD_modpain }}=1.34 \mathrm{~s}, M={ }_{\text {TFD_highpain }}=1.39 \mathrm{~s}$ ) and was significantly longer compared to dwell time on neutral faces $\left(M_{T F D \_n e u t r a l}=1.08 \mathrm{~s} ;\right.$ all $\left.F(1,36) \geq 17.51, \mathrm{p}<.0001\right)$.

To examine whether biased attention to pain at different levels of pain expressiveness is dependent upon anxiety and attention control, we first calculated total fixation bias scores for each level of pain facial expression (i.e., the degree to which participants dwelled on low, moderate, and high pain expressions in comparison to neutral faces). Using these total fixation 
bias indices, we performed a repeated measures ANCOVA with total fixation bias indices for LFE, MFE, and HFE as dependent variables and attention control and anxiety as covariates. Findings indicated a significant anxiety $\mathrm{x}$ attention control interaction $(F(, 33)=4.84, p<.05)$. No significant anxiety $\mathrm{x}$ attention control $\mathrm{x}$ facial expressiveness interaction $(F(2,32)=1.75$, ns) was observed, indicating that total fixation bias indices were not different for low, moderate, or high pain facial expressiveness. Accordingly, two univariate ANCOVAs were conducted with the mean total fixation bias (i.e., dwell time averaged across low, moderate, and high expressive pain faces) to further interpret differences for anxiety between participants who reported high levels of attention control and those who reported low levels of attention control. As shown in Figure 1, findings indicated a cross-over interaction signifying that anxiety differentially impacted the participant's dwell time on pain faces depending upon the participant's level of attention control. Specifically, for participants reporting high levels of attention control, higher anxiety was associated with increasing dwell time on pain faces. Conversely, for participants reporting low levels of attention control, higher anxiety was associated with decreasing dwell time on pain faces. While these patterns did not significantly differ from 0 for participants reporting low attention control $(F(1,33)=1.69$, ns) and only approached significance for participants reporting high attention control $(F(1,33)=3.96$, $p=.06$ ), the significant cross-over interaction indicates that these patterns are significantly different from each other. Furthermore, as shown in Figure 1 (dotted lines), findings suggest that attention control has the most impact at lower levels of anxiety. Indeed, additional analyses indicated that for participants with low levels of anxiety, increasing attention control is associated with decreasing dwell time on pain faces $(F(1,33)=9.28, p<.005)$. Attention control is not associated with dwelling on pain faces at high levels of anxiety $(F(1,33)=.002$, ns). 
Similar analysis but with pain catastrophizing entered as a covariate instead of anxiety indicated that the expected three-way interaction (i.e., catastrophizing $\mathrm{x}$ attention control $\mathrm{x}$ facial expressiveness) failed to reach significance $(F(3,31)=1.93, \epsilon=.62 ; \operatorname{NDf}(1.86 ; 19.22), \mathrm{ns})$. This suggests that the interaction between attention control and pain catastrophizing did not impact dwell time differently for neutral faces or pain faces (at any level of pain expressiveness). Analyses only revealed a significant attention control x facial expressiveness interaction $(F(3,31)=4.69, \epsilon=.62 ; \operatorname{NDf}(1.86 ; 19.22), p<.05)$ and an attention control $\mathrm{x}$ pain catastrophizing interaction $(F(1,33)=4.87, p<.05)$. The former two-way interaction (i.e., attention control $\mathrm{x}$ facial expressiveness) is not explored further since previous analyses indicated that this two-way interaction is subsumed by a three-way interaction between anxiety, attention control, and facial expressiveness.

To interpret the significant pain catastrophizing $\mathrm{x}$ attention control interaction, separate ANCOVAs were performed for participants reporting low and high attention control, respectively, and mean dwell time (averaged across the four expressiveness levels) entered as the dependent variable. As shown in Figure 2, findings indicated that pain catastrophizing was positively associated with overall dwell time (on all faces) at low levels of attention control but negatively associated with overall dwell time (on all faces) at high levels of attention control. While neither of these patterns reached significance (both $F(1,33) \leq 2.32 \mathrm{~ns}$ ), the significant cross-over interaction suggests associations are significantly different from each other. As shown in Figure 2 (dotted lines), findings suggest that attention control has the most impact at lower levels of pain catastrophizing. Indeed, additional analyses indicated that attention control is significantly positively associated with overall dwell time (i.e., to all face types) at low levels of pain catastrophizing $(F(1,33)=5.59, p<.05)$. Attention control is not associated with overall dwell time at high levels of pain catastrophizing $(F(1,33)=.38$, ns). 
In sum, eye movement analyses indicated that participants overall first fixated on pain faces more than neutral faces, and dwelled longer on pain faces than neutral faces. Whilst there was no impact of psychological variables (i.e., anxiety, pain catastrophising, or attention control) on first fixations, psychological variables did modulate dwell time on pain versus neutral faces, particularly within the context of anxiety and attention control. Specifically, anxiety differentially impacted the participant's dwell time on pain faces depending upon the participant's level of attention control. Whilst the association between pain catastrophizing and attention control was predictive of dwell time on all faces (i.e., looking for longer at all faces, regardless of face type or level of expressiveness), pain catastrophizing was not associated with biased attentional processing of pain, even when attention control was taken into account.

-INSERT FIGURE 1 ABOUT HERE-

\subsection{The impact of anxiety and pain catastrophizing upon pain outcomes, and the moderating}

$\underline{\text { role of attention control }}$

\subsubsection{Pain tolerance}

The analysis investigating the impact of anxiety and the moderating role of attention control upon the participant's pain tolerance (i.e., the participant's total immersion time during the cold pressor test) revealed a significant anxiety $\mathrm{x}$ attention control interaction $(F(1,33)=6.49, p<.05)$, indicating that the impact of anxiety upon tolerance is dependent upon the participant's level of attention control. Separate ANCOVAs for participants with high and low attention control indicated a cross-over interaction (see Figure 3). Similar to our findings on the impact of anxiety upon attentional maintenance to pain as a function of low vs. high attention control, findings indicated that the impact of anxiety upon pain tolerance shows 
opposite patterns depending upon low vs. high levels of attention control. Specifically, in line with expectations, findings indicated that higher levels of anxiety were associated with lower pain tolerance amongst participants reporting low levels of attention control $(F(1,33)=5.13$, $p<.05$ ). The opposite (but non-significant) pattern was found for participants reporting high levels of attention control $(F(1,33)=2.32$, ns). Furthermore, additional analyses (see Figure 3; dotted lines) indicated that attention control exerts opposite effects depending on whether participants are lowly or highly anxious. Specifically, for participants with low levels of anxiety, increasing attention control is associated with decreasing pain tolerance $(F(1,33)=4.02, p=.05)$. The opposite (but non-significant) pattern was found for participants reporting high levels of anxiety $(F(1,33)=3.16$, ns). The analyses with pain catastrophizing and attention control revealed no significant main or interaction effects (all $F \leq 2.40$, ns).

\subsubsection{Anticipated and experienced pain intensity}

The analyses with anxiety and attention control as independent variables and anticipated pain intensity as the dependent variable revealed a significant anxiety $\mathrm{x}$ attention control interaction $(F(1,33)=7.29, p<.05)$. Separate ANCOVAs for participants with high and low attention control indicated a crossover interaction (see Figure 4). Resembling findings on the impact of anxiety $\mathrm{x}$ attention control upon attentional maintenance to pain as well as pain tolerance, findings indicated that the impact of anxiety upon anticipated pain again shows opposite patterns depending upon low vs. high levels of attention control. Specifically, findings indicated that higher levels of anxiety were associated with higher anticipated pain amongst participants reporting low levels of attention control $(F(1,33)=9.63, p<.005)$. Findings at high levels of attention control showed the opposite pattern but failed to reach significance $(F(1,33)=.38$, ns). Furthermore, additional analyses (see Figure 4; dotted lines) indicated that attention control exerts opposite effects depending on whether participants are 
lowly or highly anxious. Specifically, for participants with low levels of anxiety, increasing attention control is associated with increasing anticipation of pain intensity $(F(1,33)=5.11$, $p<.05)$. Findings at high levels of anxiety showed the opposite pattern but failed to reach significance $(F(1,33)=3.00$, ns). The analysis with experienced pain intensity as dependent variable revealed no significant main or interaction effects (all $F(1.33)<.59$, ns).

The analyses with pain catastrophizing and attention control for both anticipated and experienced pain intensity only revealed significant main effects for pain catastrophizing such that higher levels of pain catastrophizing were associated with higher anticipated and experienced pain intensity (both $F(1.33) \geq 6.26, p<.05$ ). There were no other significant main or interaction effects (all $F(1.33) \leq 3.06$, ns).

\section{DISCUSSION}

This study examined how anxiety-related variables and attention control interacted to predict children's attention to personally relevant pain cues using eye-tracking methodology, and their tolerance of pain on the cold pressor test (CPT). Furthermore, we also explored relationships with anticipated and experienced pain intensity. Childrens' eye movements were tracked whilst viewing photographs of other children displaying painful facial expressions during the CPT, before completing the CPT themselves. We hypothesized that, particularly when attention control is low, high anxiety and pain catastrophizing would contribute to (1) increased attention towards pain cues, and (2) decreased tolerance of cold pressor pain, which can be thought of as an avoidance of further pain [8,47]. Results were in line with expectations regarding cold pressor pain outcomes, and partially in line with expectations regarding attention. Regarding attention; anxiety, pain catastrophizing, and attention control had no effect on where children first fixated their attention. That is, irrespective of anxiety or 
attention control, children tended to first fixate on pain faces. Children also tended to dwell for longer on pain faces than neutral faces (and increasingly longer on highly expressive pain faces). However, dwell time on pain faces was dependent upon anxiety, moderated by attention control. Specifically, anxiety showed opposite relationships with dwell time depending upon whether children reported low or high levels of attention control, but with findings in the opposite direction than expected. Specifically, for children reporting low levels of attention control, higher anxiety was associated with decreasing dwell time on pain faces (although children still overall attended more towards pain than neutral faces). Conversely, for children reporting high levels of attention control, higher anxiety was associated with increasing dwell time on pain faces as opposed to neutral faces. While these patterns of anxiety and pain attending did not significantly differ from zero for participants reporting low attention control, and only approached significance for participants reporting high attention control, the significant cross-over interaction indicates that these patterns are significantly different from each other. Regarding cold pressor pain outcomes; attention control played a similar moderating role. That is, at low levels of attention control, children with increasing anxiety anticipated more pain and were less tolerant for pain. The opposite pattern (though not significant in itself) was observed for children with high levels of attention control. Thus, all three analyses with anxiety and pain attending, anticipation, and tolerance showed similar cross-over interactions, indicating a robust moderating role of attention control across selfreport and behavioural outcomes (i.e., eye movements and pain tolerance). Our findings therefore suggest that when a child is increasingly anxious in the face of pain, low attention control is important in understanding worse pain outcomes. Further, our findings replicate those within the anxiety literature, demonstrating that attention control moderates the association between anxiety and attending towards threatening stimuli $[5,18,25,38,41]$, 
However, it is intriguing that our findings regarding pain attending were in the opposite direction than expected, with higher anxiety being associated with increasing dwell time on pain faces for children reporting high, not low attention control. One possible, but highly tentative explanation for these findings is that increased attending to pain cues, facilitated by high attention control, can be conceived of as a strategy employed by increasingly anxious children that may have enabled them to seek further information about the upcoming potentially painful experience, thus allowing re-appraisal. Accordingly, the observed pattern may reflect effective use of emotion regulation, in which attending to information about upcoming pain allows the regulation of aversive emotions (such as anxiety) by being able to focus on and re-appraise the threatening nature of pain. Whilst this suggestion certainly requires independent exploration, there is indeed previous evidence that adults who are highly anxious benefit from attending to rather than avoiding information about upcoming pain, such that high anxious individuals show better emotion outcomes when effortfully attending to pain (e.g., [48]). If our findings are replicated in further studies, this may suggest that the function of attention control is different for children with differing levels of anxiety. Additional analyses further attest to this notion. Specifically, we found that whilst increasing attention control was associated with better pain-related outcomes in highly anxious children (i.e., anticipating less pain and tolerating pain for longer), it was associated with poorer outcomes in lowly anxious children (i.e., anticipating more pain and tolerating pain for less time). These findings may have implications for interventions that attempt to change pain attending (e.g., Attention Bias Modification, see [17]) or more general attention control capacities (e.g., Working Memory Training, see [33]) for children with persistent pain or pain-related anxiety.

Interestingly, we found that catastrophizing about pain, unlike broader anxiety, did not interact with attention control to impact children's selective attending to pain. Catastrophizing 
did, however, interact with attention control to influence attending to all faces. Specifically, there was a cross-over interaction so that for children reporting high levels of attention control, higher catastrophising was associated with increasing dwell time on all faces. Conversely, for children reporting low levels of attention control, higher catastrophising was associated with decreasing dwell time on all faces. Nonetheless, catastrophizing did not impact selective attending to pain faces. This is somewhat in contrast with our previous findings [23], wherein catastrophizing did interact with attention control to predict adolescents' pain attending in a dot-probe task. This inconsistency may be due to the current study’s methodological advances, for example using eye-tracking and personally relevant pain cues. Importantly, children's attending to pain facial expressions that inform about an upcoming painful experience may well differ from their attending to pain faces which are not relevant to or useful for their own experience of pain [16]. Discrepancies may also be due to differences in sample ages and therefore cognitive capacities, with the current study including younger participants. Indeed, it has recently been questioned whether younger children experience truly catastrophizing thoughts, and it has instead been proposed that more general worry and anxiety is more relevant for younger children’s pain outcomes [20].

This study has limitations. First, due to technical limitations of the eye-tracker, we excluded 17 participants, resulting in a small final sample size. Of note, this may be an alternative reason for our null effects regarding pain catastrophizing. Second, eye-tracking does not measure covert attentional capture preceding saccades. Indeed, initial attention capture by threatening stimuli may occur covertly, prior to initial fixation [1]. Third, we recognize that this is a single experimental observation and replication is needed, in particular by other laboratories.

Despite these limitations, this study adds to the limited number of studies currently available on child attention and pain in three ways. 1) This is the first study to use eye- 
tracking within the context of child pain, thereby overcoming issues of using manual reaction times to infer attention, and allowing us to discriminate in real time between initial attention capture and dwelling on pain cues in youth. 2) This is one of few studies to use ecologically valid and personally salient stimuli, wherein photographs of pain facial expressions provided children with information about an upcoming personal pain experience (see also [47]). 3) This is the first study to examine the role of effortful attention control in understanding both pain attending and theoretically and clinically meaningful outcomes. Based on our findings, we believe three areas would be fruitful for further research. First, whilst the Attention Control Scale (ACS) assesses an individual's general capacity to focus in the face of distraction and to shift attention, these capacities may differ from the ability to control attention in pain-specific contexts. In particular, anxious individuals may exhibit more difficulties controlling attention in pain than non-pain contexts. The fact that we found a significant moderation effect of general attention control attests to the importance of this general capacity in influencing painspecific constructs, however, examining attention control within the context of pain may further our understanding of this effect. Second, whilst the ACS has shown good utility in predicting attentional performance in experimental tasks [18], it will be important for future studies to also include other measures of attention control such as flanker tasks (that are reliant on behavioural responses and reaction times, e.g., [21,22]) as well as antisaccade tasks (that are reliant on eye-movement patterns, e.g., [35]). These measures may be particularly important in child and adolescent samples, as brain regions engaged in goal-directed attention are still changing during this period [11,30,32,39], and so attention control may be more challenging to self-report. Third, the extent to which the reported pattern of attending is clinically relevant will rely on a demonstration of these effects in clinical samples, and a demonstration that they are malleable under instruction, practice, or therapy. 
The current findings extend our understanding of the role of child and adolescent anxiety in attending to pain cues, and anticipation and tolerance of pain. Our findings suggest that attention control is an important moderating factor for multiple outcomes relevant to these young people's pain experiences. 


\section{ACKNOWLEDGEMENTS}

The authors would like to thank Nele Decoene, Jela Van Bladel, Esther Van de Velde, Tineke Van Hove, and Laura Wyers for their assistance in recruitment and data collection. There are no conflicts of interest that may arise as a result of the research presented in this article. 


\section{FIGURE LEGENDS}

Figure 1:

(1) Mean total fixation bias to pain cues (averaged across low, moderate, and high facial pain expressiveness) as a function of low (1 SD below the mean) and high (1 SD above the mean) levels of children's anxiety and attention control

(2) Mean total fixation duration (averaged across all faces) as a function of low (1 SD below the mean) and high (1 SD above the mean) levels of children's anxiety and attention control. (3) Pain tolerance (in seconds) as a function of low (1 SD below the mean) and high (1 SD above the mean) levels of children's anxiety and attention control.

(4) Anticipated pain intensity as a function of low (1 SD below the mean) and high (1 SD above the mean) levels of children's anxiety and attention control.

All: ${ }^{* *} \mathrm{p} \leq .005 ; * \mathrm{p} \leq .05$ 


\section{REFERENCES}

[1] Armstrong T, Olatunji BO. Eye tracking of attention in the affective disorders: A metaanalytic review and synthesis. Clin. Psychol. Rev. 2012;32:704-723.

[2] Asmundson GJG, Noel M, Petter M, Parkerson HA. Pediatric fear-avoidance model of chronic pain: Foundation, application and future directions. Pain Res. Manag. 2012;17:397-405.

[3] Von Baeyer CL, Piira T, Chambers CT, Trapanotto M, Zeltzer LK. Guidelines for the cold pressor task as an experimental pain stimulus for use with children. J. Pain 2005;6:218-227.

[4] Bakker FC, Van Wieringen PCW, Van der Ploeg HM, Spielberger CD. Handleiding bij de Zelfbeoordelingsvragenlijst voor Kinderen (ZBVK). Lisse, The Netherlands: Swets \& Zeitlinger, 1989.

[5] Bardeen JR, Orcutt HK. Attentional control as a moderator of the relationship between posttraumatic stress symptoms and attentional threat bias. J. Anxiety Disord. 2011;25:1008-1018. doi:10.1016/j.janxdis.2011.06.009.

[6] Beck JE, Lipani T a., Baber KF, Dufton L, Garber J, Smith C a., Walker LS. Attentional bias to pain and social threat in pediatric patients with functional abdominal pain and pain-free youth before and after performance evaluation. Pain 2011;152:1061-1067. doi:10.1016/j.pain.2011.01.029.

[7] Birnie K a, Caes L, Wilson AC, Williams SE, Chambers CT. A practical guide and perspectives on the use of experimental pain modalities with children and adolescents. Pain Manag. 2014;4:97-111. doi:10.2217/pmt.13.72.

[8] Boston A, Sharpe L. The role of threat-expectancy in acute pain: Effects on attentional bias, coping strategy effectiveness and response to pain. Pain 2005;119:168-175.

[9] Boyer MC, Compas BE, Stanger C, Colletti RB, Konik BS, Morrow SB, Thomsen AH. Attentional biases to pain and social threat in children with recurrent abdominal pain. J. Pediatr. Psychol. 2006;31:209-220.

[10] Chambers CT, McGrath PJ, Gilbert CA, Craig KD. Child facial coding system revised manual. Halifax, NS, Canada: IWK-Grace Health Centre, Dalhousie University \& University of British Columbia, 1996.

[11] Cohen Kadosh K, Heathcote LC, Lau JYF. Age-related changes in attentional control across adolescence: how does this impact emotion regulation capacities? Front. Psychol. 2014;5:111. doi:10.3389/fpsyg.2014.00111.

[12] Crombez G, Bijttebier P, Eccleston C, Mascagni T, Mertens G, Goubert L, Verstraeten $\mathrm{K}$. The child version of the pain catastrophizing scale (PCS-C): A preliminary validation. Pain 2003;104:639-646. 
[13] Crombez G, Eccleston C, Baeyens F, Eelen P. When somatic information threatens, catastrophic thinking enhances attentional interference. Pain 1998;75:187-198.

[14] Van Damme S, Crombez G, Eccleston C. Disengagement from pain: the role of catastrophic thinking about pain. Pain 2004;107:70-76. doi:http://dx.doi.org/10.1016/j.pain.2003.09.023.

[15] Van Damme S, Legrain V, Vogt J, Crombez G. Keeping pain in mind: A motivational account of attention to pain. Neurosci. Biobehav. Rev. 2010;34:204-213.

[16] Van Damme S, Legrain V, Vogt J, Crombez G. Keeping pain in mind: A motivational account of attention to pain. Neurosci. Biobehav. Rev. 2010;34:204-213.

[17] Dehghani M, Sharpe L, Nicholas MK. Modification of attentional biases in chronic pain patients: a preliminary study. Eur J Pain 2004;8:585-594.

doi:10.1016/j.ejpain.2004.02.003.

[18] Derryberry D, Reed MA. Anxiety-related attentional biases and their regulation by attentional control. J. Abnorm. Psychol. 2002;111:225-236.

[19] Eccleston C, Crombez G. Pain demands attention: a cognitive-affective model of the interruptive function of pain. Psychol. Bull. 1999;125:356-366.

[20] Eccleston C, Fisher EA, Vervoort T, Crombez G. Worry and catastrophizing about pain in youth: A reappraisal. Pain 2012;153:1560-1562.

[21] Eriksen BA, Eriksen CW. Effects of noise letters upon the identification of a target letter in a nonsearch task. Percept. Psychophys. 1974;16:143-149.

[22] Fan J, McCandliss BD, Sommer T, Raz A, Posner MI. Testing the efficiency and independence of attentional networks. J. Cogn. Neurosci. 2002;14:340-347.

[23] Heathcote LC, Vervoort T, Eccleston C, Fox E, Jacobs K, Ryckeghem DML Van, Lau JYF. Relationship between adolescents ' pain catastrophizing and attention bias to pain faces is moderated by attention control. Pain n.d.

[24] Heathcote LC, Vervoort T, Eccleston C, Fox E, Jacobs K, Van Ryckeghem DML, Lau JYF. The relationship between adolescents' pain catastrophizing and attention bias to pain faces is moderated by attention control. Pain 2015;156:1334-1341. doi:10.1097/j.pain.0000000000000174.

[25] Helzer EG, Connor-Smith JK, Reed M a. Traits, states, and attentional gates: temperament and threat relevance as predictors of attentional bias to social threat. Anxiety. Stress. Coping 2009;22:57-76.

[26] Holmbeck GN. Post-hoc probing of significant moderational and mediational effects in studies of pediatric populations. J. Pediatr. Psychol. 2002;27:87-96. 
[27] King S, Chambers CT, Huguet A, MacNevin RC, McGrath PJ, Parker L, MacDonald AJ. The epidemiology of chronic pain in children and adolescents revisited: A systematic review. Pain 2011;152:2729-2738. doi:10.1016/j.pain.2011.07.016.

[28] Komogortsev O V., Gobert D V., Jayarathna S, Koh DH, Gowda SM. Standardization of automated analyses of oculomotor fixation and saccadic behaviors. IEEE Trans. Biomed. Eng. 2010;57:2635-2645.

[29] Meesters C, Muris P, Van Rooijen B. Relations of neuroticism and attentional control with symptoms of anxiety and aggression in non-clinical children. J. Psychopathol. Behav. Assess. 2007;29:149-158.

[30] Monk CS, McClure EB, Nelson EE, Zarahn E, Bilder RM, Leibenluft E, Charney DS, Ernst M, Pine DS. Adolescent immaturity in attention-related brain engagement to emotional facial expressions. Neuroimage 2003;20:420-428.

[31] Muris P, Van Der Pennen E, Sigmond R, Mayer B. Symptoms of anxiety, depression, and aggression in non-clinical children: Relationships with self-report and performance-based measures of attention and effortful control. Child Psychiatry Hum. Dev. 2008;39:455-467.

[32] Nelson CA, Bloom FE, Cameron JL, Amaral D, Dahl RE, Pine D. An integrative, multidisciplinary approach to the study of brain-behavior relations in the context of typical and atypical development. Dev. Psychopathol. 2002;14:499-520.

[33] Owens M, Koster EHW, Derakshan N. Improving attention control in dysphoria through cognitive training: Transfer effects on working memory capacity and filtering efficiency. Psychophysiology 2013;50:297-307.

[34] Roelofs J, Peters ML, van der Zijden M, Thielen FGJM, Vlaeyen JWS. Selective attention and avoidance of pain-related stimuli: a dot-probe evaluation in a pain-free population. J. Pain 2003;4:322-328. doi:http://dx.doi.org/10.1016/S15265900(03)00634-5.

[35] Rommelse NNJ, Van der Stigchel S, Sergeant JA. A review on eye movement studies in childhood and adolescent psychiatry. Brain Cogn. 2008;68:391-414.

[36] Van Ryckeghem DML, Crombez G, Van Hulle L, Van Damme S. Attentional bias towards pain-related information diminishes the efficacy of distraction. Pain 2012;153:2345-2351. doi:10.1016/j.pain.2012.07.032.

[37] Salvucci DD, Salvucci DD, Goldberg JH, Goldberg JH. Identifying Fixations and Saccades in Eye-Tracking Protocols. Proc. Eye Track. Res. Appl. Symp. 2000:71-78.

[38] Schoorl M, Putman P, Van Der Werff S, Van Der Does a. JW. Attentional bias and attentional control in Posttraumatic Stress Disorder. J. Anxiety Disord. 2014;28:203210. doi:10.1016/j.janxdis.2013.10.001.

[39] Spear LP. The adolescent brain and age-related behavioral manifestations. Neurosci. Biobehav. Rev. 2000;24:417-463. 
[40] Spielberger CD, Edwards CD, Lushene RE, Montuori J, Platzek D. The State-Trait Anxiety Inventory for Children (preliminary manual). Palo Alto, CA: Consulting Psychologists Press, 1973.

[41] Susa G, Pitică I, Benga O, Miclea M. The self regulatory effect of attentional control in modulating the relationship between attentional biases toward threat and anxiety symptoms in children. Cogn. Emot. 2012;26:1069-1083.

[42] Verhoeven K, Crombez G, Eccleston C, Van Ryckeghem DML, Morley S, Van Damme S. The role of motivation in distracting attention away from pain: An experimental study. Pain 2010;149:229-234. doi:http://dx.doi.org/10.1016/j.pain.2010.01.019.

[43] Vervoort T, Caes L, Crombez G, Koster E, Van Damme S, Dewitte M, Goubert L. Parental catastrophizing about children's pain and selective attention to varying levels of facial expression of pain in children: A dot-probe study. Pain 2011;152:1751-1757.

[44] Vervoort T, Caes L, Trost Z, Notebaert L, Goubert L. Parental attention to their child's pain is modulated by threat-value of pain. Heal. Psychol. 2012;31:623-631.

[45] Vervoort T, Goubert L, Crombez G. The relationship between high catastrophizing children's facial display of pain and parental judgment of their child's pain. Pain 2009;142:142-148.

[46] Vervoort T, Trost Z, Prkachin KM, Mueller SC. Attentional processing of other's facial display of pain: An eye tracking study. Pain 2013;154:836-844.

doi:10.1016/j.pain.2013.02.017.

[47] Vervoort T, Trost Z, Van Ryckeghem DML. Children's selective attention to pain and avoidance behaviour: The role of child and parental catastrophizing about pain. Pain 2013;154:1979-1988. doi:10.1016/j.pain.2013.05.052.

[48] Vervoort T, Trost Z, Sütterlin S, Caes L, Moors A. Emotion regulatory function of parent attention to child pain and associated implications for parental pain control behaviour. Pain 2014;155:1453-1463. doi:10.1016/j.pain.2014.04.015.

[49] Vlaeyen JWS, Linton SJ. Fear-avoidance and its consequences in chronic musculoskeletal pain: A state of the art. Pain 2000;85:317-332. 